\title{
Hercules X-1 (Her X-1): New results from the past decade of observations
}

\section{Denis A. Leahy ${ }^{a, *}$}

${ }^{a}$ Department of Physics and Astronomy, University of Calgary 2500 University Dr. NW, Calgary, Canada

E-mail: leahy@ucalgary.ca

Hercules $\mathrm{X}-1$ is an accreting X-ray binary with well-determined properties based on measurements of light-curves and spectra since its discovery in 1972. Since discovery, it has been observed with most major X-ray astronomy instruments. Analyses of Her X-1 over the past decade include, among others, observations from RXTE/PCA and ASM, Suzaku, NuSTAR, AstroSat and Swift/BAT. These have added to the range of phenomena detected from this system. New aspects of the binary are reviewed. The data are being used to test models for the system, including the nature of the companion star (HZ Her), the accretion column on the neutron star, the shape of the accretion disk and its shadow, and the accretion stream.

*** High Energy Astrophysics in Southern Africa (HEASA2021) ***

*** 13 - 17 September, $2021 * * *$

$* * *$ Online $* * *$

${ }^{*}$ Speaker 


\section{Introduction}

Hercules X-1 is a bright X-ray binary which was discovered early (1972) in the history of $\mathrm{X}$-ray astronomy [1]. Here we summarize the highlights of previous work illuminating the nature of the X-ray binary. The discovery paper [1] reported $1.24 \mathrm{~s} \mathrm{X-ray} \mathrm{pulsations} \mathrm{from} \mathrm{the} \mathrm{rotating}$ neutron star and measured a $1.7 \mathrm{~d}$ orbital period from Doppler time delays. Further observations with UHURU [2] revealed a 35-day repeating cycle of "on" and "off" states for which the flux was bright and faint, respectively, as well as the light curve for the "on" states which exhibits a rapid (few hour) rise of flux, followed by a slow (several days long) decline of flux.

An early explanation for the 35-day cycle was that it was driven by precession of the companion star (HZ Her) which had its rotation axis tilted with respect to the binary axis [3]. Thus the accretion stream from the companion would be in a plane tilted at an angle from the binary equatorial plane. The accretion stream plane would rotate at the precession period of the companion, producing a twisted accretion disk. This twisted disk rotates at the companion's precession period, thus providing a 35-day cycle during which the neutron star would be blocked from view by the disk for roughly half the period (Figure 2 in [3]). However, a consistent explanation of the physical mechanism causing the twisted disk was not obtained until the radiation-driven model of [4]. Almost at the same time, long term observations of the 35-day cycle gave the first measurements of the average shape of the 35-day light-curve [5]. The modelling study of [6] utilized that data to infer the shape of the twisted accretion disk.

Observations of the optical radiation from the companion star HZ Her over a 35-day cycle [7] was the next break-through in understanding the shape of the accretion disk. The optical light-curve is mainly driven by the X-ray illumination, and subsequent heating, of the face of HZ Her which faces the neutron star. This makes the illuminated part of the HZ Her much brighter than it would otherwise be with a temperature $\sim 16,000 \mathrm{~K}$ for the heated surface compared to $\sim 7000 \mathrm{~K}$ for the unheated surface. The moving X-ray shadow cast by the precessing tilted accretion disk results in the shape of the optical light-curve. Thus the optical light-curve provides clear evidence that of the existence of such a disk. [8] analyzed UHURU observations of Her X-1 during the $~ 22$-day long "off" state and discovered a secondary peak in X-ray emission, at $\sim 20 \%$ of the intensity of the main "on" state, which lasts $\sim 5$ days. The main "on" state is now known as the Main High state and the secondary "on" state is known as the Short High state, while the two very low intensity intervals, between Main High and Short High and between Short High and the following Main High, are known as the Low States.

In addition to the 35-day cycle, the light-curve exhibits frequent and regular short and strong decreases, called dips. The first comprehensive study of dips was by [9]. A steady shift of orbital phase of dips with 35-day phase (the marching dips phenomenon) was found. The first spectral study of dips, which are observed during Main High, was presented by [10] using the spectral and time resolution enabled by GINGA observations. High signal-to-noise spectra were obtained with observation times between $\sim 30$ and $200 \mathrm{~s}$. The partial covering model for the spectrum was shown to fit the Main High spectrum of Her X-1. The Main High spectrum is dominated by X-rays between $\sim 2$ and $20 \mathrm{keV}$, with significantly less flux outside that band. During dips the main change was the increase of cold matter column density, accompanied by changes in fluorescent iron intensity. [11] presented a comprehensive study of dips observed in Her X-1 by GINGA. In addition to Main 
High state dips, dips during Short High were measured. Dip durations were shown to be highly variable, from $\sim 1$ minute to 6 hours. The column density of dips was found to show a clear increase with orbital phase. More comprehensive studies of dips were carried out using RXTE/PCA data. [12] analyzed the frequency of dips vs. orbital and 35-day phases, finding strong dependence on orbital phase, with Main High affected by dips for 30\% of the time, and Short High 75\%. [13] characterized 370 individual dips with durations of $32 \mathrm{~s}$ to $15 \mathrm{hr}$.

Further clues on the X-ray binary and its disk were obtained from analysis of the energy dependence of the X-ray pulsations [14], observed at the rotation period (1.24 s) of the neutron star. At energies below $1 \mathrm{keV}$, the pulsations are smoother and out of phase by $\sim 180^{\circ}$ with respect to the higher energy pulsations. This is evidence that the low energy $\mathrm{X}$-rays are reprocessed by the inner edge of the accretion disk: the far side of the inner edge is visible to the observer and it is bright at $<1 \mathrm{keV}$ when the $>1 \mathrm{keV} \mathrm{X}$-ray beam from the neutron star is pointing away from us $\left(180^{\circ}\right.$ in pulse phase after the $>1 \mathrm{keV} \mathrm{X-ray} \mathrm{beam} \mathrm{is} \mathrm{pointing} \mathrm{toward} \mathrm{the} \mathrm{observer).} \mathrm{The} \mathrm{full} \mathrm{nature} \mathrm{of} \mathrm{both}$ the 35-day dependence of the pulse shape, and the pulse shape dependence on X-ray energy were revealed by the study of [15]. That work conclusively showed that both the 35-day light-curve and the 35-day cycle in pulse shape were caused by the precessing accretion disk.

The first report of an extended (months-long) period of weak emission from Her X-1, called an anomalous low state or ALS, was by [16]. This was suggested to be caused by a temporary change in disk structure, which was later confirmed by disk model calculations by [17].

The accurate measurement of the broad-band X-ray spectrum of Her X-1 had to await the launch of the BeppoSAX broad-band X-ray observatory. [18] show that the spectrum has multiple components: a double power law with power-law break of $\sim 18 \mathrm{keV}$ plus exponential cutoff (with $E_{\text {fold }}=15 \mathrm{keV}$ and $E_{\text {cut }}=24 \mathrm{keV}$ ); a low energy black-body with temperature $\sim 0.1 \mathrm{keV}$; broad (0.4 keV FWHM) emission near $1 \mathrm{keV}$ assigned to the Fe L complex of emission lines; Fe K-line emission near $6.5 \mathrm{keV}$; and superimposed broad ( $\sim 15 \mathrm{keV}$ FWHM) cyclotron absorption line at 40 $\mathrm{keV}$. The spectrum indicates that several emission regions contribute to the X-ray emission: hard $\mathrm{X}$-rays from the accretion column, with the cyclotron absorption line, corresponding to a field of $\sim 3 \times 10^{12}$ Gauss originating from the magnetic field near the neutron star surface and co-located with the column; black-body emission and Fe L-line emission from the heated inner edge of the accretion disk; and fluorescent Fe K-line emisson from the inner edge of the accretion disk and from the accretion stream incoming to the accretion column.

\section{New Results in the Past 10 Years}

Here we present highlights of the new results on the nature of the X-ray binary Her X-1 in chronological order. This summary is not complete but is meant to represent the most important results on the physical nature of Her X-1 in the past decade.

The likely-correct model for dips was presented and calculated by [19], which is the impact point of the accretion stream and the disk, which periodically moves into the line-of-sight from observer to the neutron star. Further spectral observations with better instruments have improved the measurement of the spectrum and included partial covering absorption (e.g. [20]). That the precessing accretion disk produces both the 35-day light-curve and the 35-day cycle in pulse shape changes was confirmed by [21]. 
Accurate RXTE/PCA eclipse ingress and egress timings were analyzed by [22] to derive the radius of $\mathrm{HZ} \mathrm{Her} \mathrm{(to} \sim 2$ parts in 1000). The uncertainty in velocity amplitude of HZ Her ( $\sim 10 \%)$ and uncertainty in system inclination $(\sim 5 \%)$ dominate the resulting uncertainty in masses of the neutron star $\left(1.50 \mathrm{M}_{\odot}\right)$ and of $\mathrm{HZ} \operatorname{Her}\left(2.33 \mathrm{M}_{\odot}\right)$. The evolutionary state and distance to Her X-1 were found by analysis of the optical spectrum of the unheated side of HZ Her combined with stellar evolution calculations.

The Low and Short High state light-curves and spectra were analyzed by [23] using RXTE/PCA observations to obtain the best sensitivity yet attained. They showed the low-state flux is dominated by X-ray reflection off of HZ Her, and that the short high flux has a reflection contribution plus a larger orbital-phase-independent contribution, which can be attributed to scattered X-ray flux. The density and shape of the extended scattering corona surrounding Her X-1 was measured using RXTE/PCA measurements of eclipses by [24].

[25] applied their physically-motivated "radiation-dominated radiative shock model for Comptonized accretion flows" to NuSTAR observations of Her X-1 during Main High. They derived Comptonizing temperature, radius and average scattering opacity in the accretion column, and the accretion rate from the spectrum, for the first time. An additional component, a highly ionized absorber, was detected in the X-ray spectrum during Main High by [26]. This ionized wind/corona, its outflow velocity and mass loss rate were measured using absorption lines of N VII, O VIII and $\mathrm{Ne} X$ with XMM-Newton [27]. The behaviour of the well-known cyclotron line over the past 20 years was summarized by [28]: it shows a slow secular decline with shorter time-scale variations.

AstroSAT/UVIT observations in the FUV band are near-ideal for studying the X-ray heated face of HZ Her. [29] measured the 148nm FUV orbital light-curve during Main High and showed that it was well modeled by two components: a part which is caused by the changing view of the $\mathrm{X}$-ray heated face of HZ Her with orbital phase; and a part which is slowly changed from the inner region of the accretion disk. A fit to the light-curve gave the best-yet constraints on the accretion disk (tilt and twist angles). The 35-day cycle has been thoroughly characterized using $\sim 15$ years of observations each with Swift/BAT and RXTE/ASM by [30].

\section{Summary and Conclusion}

This short review of the Her X-1 X-ray binary is meant to serve as a guide to the main results, historical (in the Introduction) and in the past decade (Section 2). This system, discovered early in the history of X-ray astronomy, has been an important relatively nearby and un-obscured X-ray binary which is accessible to observations in many wavebands: infrared, optical, UV and X-rays. The multi-wavelength view has allowed a wide variety of studies to allow us to deduce the various components in exquisite detail: the companion star (HZ Her), the accretion disk and its corona, and the accretion flow/accretion column on the neutron star. In the near future, there is promise to learn about the accretion stream from studies the light-curve dips.

Her X-1 is likely to continue to serve as a model system for studies of X-ray binaries.

\section{Acknowledgments}

This work supported by the Natural Sciences and Engineering Research Council of Canada. 


\section{References}

[1] Tananbaum, H., Gursky, H., Kellogg, E. M., et al. Discovery of a Periodic Pulsating Binary X-Ray Source in Hercules from UHURU, Astrophys. J. Lett. 1972, 174, L143.

[2] Giacconi, R.; Gursky, H.; Kellogg, E.; Levinson, R.; Schreier, E.; Tananbaum, H. Further X-ray observations of Hercules X-1 from Uhuru, Astrophys. J. 1973, 184, 227-236.

[3] Petterson, J.A. Hercules X-1: A neutron star with a twisted accretion disk?, Astrophys. J. 1975, 201, L61-L64.

[4] Wijers, R.A.M.J; Pringle, J.E. Warped accretion discs and the long periods in X-ray binaries. Mon. Not. R. Astron. Soc. 1999, 308, 207-220.

[5] Scott, D.M; Leahy, D.A. Rossi X-Ray Timing Explorer All-Sky Monitor Observations of the 35 Day Cycle of Hercules X-1. Astrophys. J. 1999, 510, 974-985.

[6] Leahy, D.A. Modelling RXTE/ASM observations of the 35-d cycle in Her X-1. Mon. Not. R. Astron. Soc. 2002, 334, 847-854.

[7] Gerend, D; Boynton, P.E. Optical clues to the nature of Hercules X-1 /HZ Herculis. Astrophys. J. 1976, 209, 562-573.

[8] Jones, C. \& Forman, W. 1976, Uhuru observations of Hercules X-1 during the low state of the 35-day cycle. Astrophys. J. Lett. 1976, 209 L131.

[9] Crosa, L. \& Boynton, P. E. Periodic mass transfer in HER X-1/ HZ Her. Astrophys. J. 1980, $235,999$.

[10] Leahy, D.A.; Yoshida, A.; Matsuoka, M. Spectral Evolution during Pre-Eclipse Dips in Hercules X-1. Astrophys. J. 1994, 434, 341-347.

[11] Leahy, D.A. GINGA observations of absorption dips in Hercules X-1. Mon. Not. R. Astron. Soc. 1997, 287, 622-628.

[12] Leahy, D.A; Igna, C. The Light Curve of Hercules X-1 as Observed by the Rossi X-Ray Timing Explorer. Astrophys. J. 2011, 736, 74-80.

[13] Igna, C.D; Leahy, D.A. Hercules X-1's light-curve dips as seen by the RXTE/PCA: A study of the entire 1996 February-2005 August light-curve. Mon. Not. R. Astron. Soc. 2011, 418, 2283-2291.

[14] McCray, R. A., Shull, J. M., Boynton, P. E., et al. Einstein Observatory pulse-phase spectroscopy of HER X-1. Astrophys. J. 1982, 262,301.

[15] Scott, D.M.; Leahy, D.A.; Wilson, R.B. The 35 Day Evolution of the Hercules X-1 Pulse Profile: Evidence for a Resolved Inner Disk Occultation of the Neutron Star. Astrophys. J. 2000, 539, 392-412. 
[16] Parmar, A.N.; Pietsch, W.; McKechnie, S.; White, N.E.; Truemper, J.; Voges, W.; Barr, P. An extended X-ray low state from Hercules X-1. Nature 1985, 313, 119-121.

[17] Leahy, D. A. \& Dupuis, J., Extreme Ultraviolet Explorer Observations of Hercules X-1 over a 35 Day Cycle Astrophys. J. 2010715897.

[18] dal Fiume, D.; Orlandini, M.; Cusumano, G.; Del Sordo, S.; Oosterbroek, T.; Cusumano, G.; Parmar, A.N.; Santangelo, A.; Feroci, M.; Frontera, F.; et al. The broad-band (0.1-200 keV) spectrum of HER X-1 observed with BeppoSAX. Astron. Astrophys. 1998, 329, L41-L44.

[19] Igna, C.D; Leahy, D.A. Light-curve dip production through accretion stream-accretion disc impact in the HZ Her/Her X-1 binary star system. Mon. Not. R. Astron. Soc. 2012, 425, 8-20.

[20] Asami, F., Enoto, T., Iwakiri, W., et al. Broad-band spectroscopy of Hercules X-1 with Suzaku, PA.S.J. 2014, 66, 44.

[21] Staubert, R.; Klochkov, D.; Vasco, D.; Postnov, K.; Shakura, N.; Wilms, J.; Rothschild, R.E. Variable pulse profiles of Hercules X-1 repeating with the same irregular $35 \mathrm{~d}$ clock as the turn-ons. Astron. Astrophys. 2013, 550, 110-118.

[22] Leahy, D.A; Abdallah, M.H. HZ Her: Stellar Radius from X-Ray Eclipse Observations, Evolutionary State, and a New Distance. Astrophys. J. 2014, 793, 79-86.

[23] Abdallah, M.H; Leahy, D.A. Spectral signature of atmospheric reflection in Hercules X-1/HZ Hercules during low and short high states. Mon. Not. R. Astron. Soc. 2015, 4, 4222-4231.

[24] Leahy, D. A. 2015, Hercules X-1: Using Eclipse to Measure the X-Ray Corona. Astrophys. J. 2015, 800, 32 .

[25] Wolff, M. T., Becker, P. A., Gottlieb, A. M., et al. The NuSTAR X-Ray Spectrum of Hercules X-1: A Radiation-dominated Radiative Shock. Astrophys. J. 2016, 831, 194.

[26] Leahy, D.A; Chen, Y. AstroSat SXT Observations of Her X-1. Astrophys. J. 2019, 871, $152-161$.

[27] Kosec, P., Fabian, A. C., Pinto, C., et al. An ionized accretion disc wind in Hercules X-1 Mon. Not. R. Astron. Soc. 2020, 491, 3730.

[28] Bala, S., Bhattacharya, D., Staubert, R., et al. 2020, Time evolution of cyclotron line of Her X-1: a detailed statistical analysis including new AstroSat data. Mon. Not. R. Astron. Soc. 2020, 497, 1029.

[29] Leahy, D.A.; Postma, J.; Chen, Y. AstroSat UVIT Observations of Her X-1. Astrophys. J. 2020, 889, 131-139.

[30] Leahy, D; Wang, Y. Swift/BAT and RXTE/ASM Observations of the 35 day X-Ray Cycle of Hercules X-1. Astrophys. J. 2020, 902, 146-154. 